\title{
The Constructing of Semantic Frame and the Conceptual Integration of Translation Process
}

\author{
Gao-pan GUO ${ }^{1, a,{ }^{*}}$ and Zong-ying WANG ${ }^{2}$ \\ 1,2East China University of Technology, Nanchang, Jiangxi, China \\ aguogaopan@126.com
}

\begin{abstract}
Keywords: FrameNet, Semantic Frame, Translation Teaching, Conceptual
\end{abstract} Integration.

\begin{abstract}
The translation process is the process of conceptual frame shift and the semantic shifting. This article uses "FrameNet" corpus to describe the constructing process of semantic frame, to uncover the basic structure of semantic information and valence patterns with the concept of Frame and syntactic realizations for the psychological understanding of translation. Meanwhile, with the help of conceptual mapping and conceptual integration, this research means to lessen the negative effect produced by translator's subjective judgment on the production of translation, to strengthen the operation and explanation of concept shifting during the translation teaching process.
\end{abstract}

\section{Introduction}

Cognitive linguistics has its theoretical foundation and takes its inspiration from psychology and philosophy that emphasize the importance of human experience. According to this empiricist view, the centrality of language, its meaning, originates from the nature of human bodies or human experience and has its significance because of the interaction with the world. The meaning of language is not carried by the packaged self-contained units with different forms, but "is prompted by words and these prompts serve as instructions for conceptual processes that result in meaning construction" [1]. The conceptual representations are integrated with different conceptual mapping relations in a certain frame. Due to the differences in the economic, cultural and customs of each ethnic group, the process of translation is not the transformation of the conceptual equivalence between two languages, but embodies the emergent conceptual meaning with the psychological characteristics of selectivity, iconicity, analogy, etc. The nature of the process of translation is relatively complex, then how to interpret and present the conceptual framework of the construction and semantic positioning during the translation teaching process? The purpose of this study, with the frame concept in cognitive corpus "FrameNet", is to explore the basic structure of semantic information storage and application in the human brain and to form a comprehensive impression of the psychological aspects of translation. In this way, we tend to reduce the negative impact of translator's the subjective judgments on the concept of translation process, and to facilitate the translation teaching with more technical basis and dynamic operations.

\section{FrameNet and Frame Semantics}

Cognitive corpus project "FrameNet" is founded by American national Science Found (https://framenet.icsi.berkeley.edu/fndrupal/). This corpus aims to create a lexical database of English that is both human- and machine-readable, based on annotating examples of how words are used in actual texts. By providing semantic and syntactic 
universalization corpus evidence, target words and phrases' valence expression, the semantic framework of word items is described in the corpus. FrameNet exemplifies all the frame elements and their syntax realization in the aspect of grammatical function, lexical type, and syntax characters.

The FrameNet team has defined more than 1,000 semantic frames and has linked them together by a system of frame relations. This corpus is also supported by the British National Corpus (BNC) with the annotated semantic information. The FrameNet corpus has a traditional theoretical foundation "Frame Semantics" created by Charles Fillmore and his researching team [2,3]. Fillmore points out that this theory has well explained the meaning constructing process of the lexical and sentence construction. During the communication and reading, the abstract dictionary explanation alone is hard to explain the difficult semantic relations, because the speaker or reader has a good understanding of the semantic frame which is prompted by words with their body or psychological experience. This semantic frame concludes the described event, relations, entity and the participants. For instance, the conceptual frame of "cooking" typically involves some frame elements, such as a person doing the cooking (Cook), the food that is to be cooked (Food), something to hold the food while cooking (Container) and a source of heat (Heating instrument). This corpus aims to construct and define different frames in the conceptual structures, and also demonstrate the conceptual frames and its related frame elements in the grammatical construction.

Take a sentence for example:

[Cook The boys] GRILL [Food their fishes] [Heating instrument on an open fire].

In this simple conceptual frame, the word "GRILL" triggers the emergence of the frame, and the frame elements construct the syntactic argument structure. That is "the boys" is the subject of GRILL, "their fishes" is the direct object and "on an open fire" is the prepositional phrase modifying the prompt GRILL.

\section{The Operational Process of Semantic Frame Construction and the Conceptual Transformation of Translation}

The nature of translation process is the transformation of conceptual frame and the semantic reposition. The conceptual frames prompted by the words or multi-word units are not completely the same among different languages. The meaning emergence in a frame and the frame relations are the activation and integration of the related cognitive domain, knowledge frame, cultural background and etc. while the meaning emergence in the conceptual frame is a complex and multifactor related process, for the translation learners, it will be a big challenge for them to understand the conceptual transformation and semantic positioning in the different asymmetric frame.

The frame concept is based on the psychological processing and conceptual integration of the describable information. This information integrates into information packets which constructs the frame net with different frame relations. The constructing mode of frame net is similar with the conceptual integration process in translation, and it's a very important base for improving the quality of translation [4]. The following example will demonstrate the practical operation process of conceptual transformation and translation understanding.

For example: John replaced me.

Words are prompts for meaning construction rather than "containers" that carry meaning [5]. In this sentence, the verb "replace" triggers the emergence of frame and 
reflects the relationship with the frame elements "John" and "me". When the verb "replace" is searched in the FrameNet corpus for frame description, the result shows two frames, "Take place of" and "Replacing", which are defined as "take the place of" and "to put something new in the place of something" respectively (see figure 1). Generally, there are some frame elements in a frame, including "agent", "explanation", "manner", "means", "new", "old", "place", "purpose", "time". In this example, the frame elements "agent", "new", and "old" are taken for explanation.

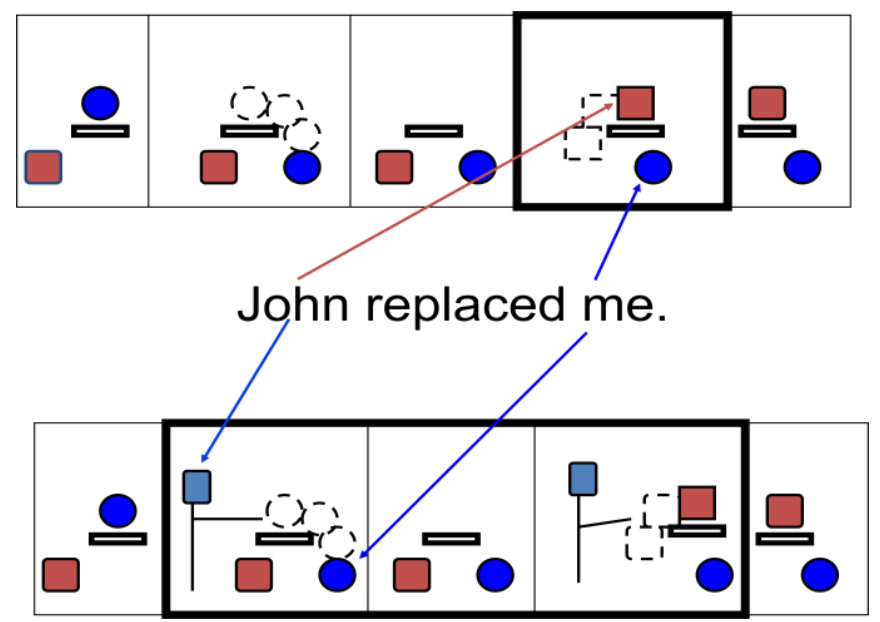

Figure 1. the relationship between a frame and frame elements (adapted from https://framenet.icsi.berkeley.edu/fndrupal/)

During the process of translation, if the concept in the frame is interpreted as syntagmatic processing method, the sentence will be translated as:

$$
\begin{aligned}
& \text { John replaced me. (English) } \\
& \text { 约翰代替了我。 } \\
& \text { (Chinese) }
\end{aligned}
$$

However, this translation has only expressed one aspect of the English sentence meaning and missed the other one: John replaced me with someone new in the place of my position rather than himself. With the help of the FrameNet corpus, the frame elements are clearly described and it also shows the valence and syntactic relation:

[New John] REPLACED ${ }^{\text {Target }}$ [old me].

[Agent John] REPLACED ${ }^{\text {Target }}$ [old me].

Based on the clear valence relation, the translation can be carried out in a logical reasoning process, integrating with the formal schemata and sociocultural knowledge. This process can effectively avoid translation difficulties caused by polysemy, semantic ambiguity and unclear context. At the same time, it also reflects the multi-level relation of sentence meaning and provides a good basis for semantic choice.

\section{The Operational Translation Teaching Process}

Generally, the difficult part for teaching translation is how to present the abstract concepts of logical reasoning, schema structure, conceptual integration, and semantic choice to the learners, which leads to the over-reliance on grammar analysis and sentence partition. Thereby it enhances the learners' dependence on meaning equivalence when dealing with translation. While the FrameNet corpus uses the valence relation among the framework elements to realize the process of logic 
processing, and shows the way of constructing among information packages, which makes it possible to analyze conceptual transformation and conceptual integration of translation process. The following translation examples are partly taken from English-Chinese translation passages in Chinese Test for English Majors-Band 8 (TEM8), in 2015.

\section{Example 1: At Its Heart, Psycholinguistic Work Consists of Two Questions}

In the constructing of meaning, the prepositional phrase "At its heart" is a space builder that sets up a base space in the mental space. In this space, there are two space elements, "psycholinguistic work" and "questions". With the schema induction, the frame concept constructed by the verb phrase "consists of" reflects the relationship between the two space elements. With the help of the anaphor "its", the whole sentence can be rewritten as:

Heart of Psycholinguistic Work Consists of Two Questions. The conceptual metaphor triggered by "heart" is a very popular phenomenon during the translation process. "metaphor" is one of the top ten hot phrase in Webster Dictionary published in 2013. Naturally, we can understand the abstract and unreadable concept with the help of the real and concrete item's characters. However, if the conceptual metaphor is taken as a tool of cognitive analysis, its shortage is clear, and leads to the partial understanding of a concept.

The FrameNet corpus has not covered all the words with frame description, but it has provided over 170, 000 manual annotated sentences which can give us unique semantic analysis data. The emergent meaning of metaphor during the integration is unlimited theoretically, but the formal expressions and the ways of sentence realization are limited. So when the word "heart" in the example is searched in the FrameNet corpus, all the syntactic relations constructing in the frame, with one exception, is expressed as "heart of NP (Noun Phrase)", which is defined as "vital part of something". We take this common frame construction in the meaning deconstruction and sentence translation. The translation follows as:

$$
\begin{aligned}
& \text { Heart of psycholinguistic work... (English) } \\
& \text { 心理语言学最重要的研究... (Chinese) }
\end{aligned}
$$

The verb phrase "consists of" in the frame is defined as "make up of". According to Talmy's "Attentional System" in "Conceptual Structuring System Model" [6], the focus of the "consists of" frame is "figure" not the "background", that is the frame element "psycholinguistic work" but not the "questions". In order to avoid the absolute equivalence between the source conceptual structure and the target conceptual structure, the whole sentence can translate as:

At its heart, psycholinguistic work consists of two questions. (English) 心理语言学最重要的研究包括两个问题。

(Chinese)

Example 2: One is, What Knowledge of Language is Needed for Us to Use Language?

Based on the anaphor "one" and textual background constructing by the above sentence, it reveals that this sentence has not built the new mental space.

In this corpus, verb "need" triggers the frame "Needing", while "needed" triggers the frame "Being necessary". The translation expression will be different when we use 
different frames. However, the way of meaning constructing and the characters of syntax realization in the FrameNet can give us a good choice during the translation process. When the frame elements are well explained by the valence relations, it is reasonable to have a translation with Chinese character.

[Dependent What knowledge of language] is NEEDED ${ }^{\text {Target }}$ [Cognizer for us] [Requirement to use language]?

According to the syntax realization and valence relation, the logical relation of this sentence is revealed in the frame "Needing". The cognizer of the frame element is "us" but not the "knowledge of language". This sentence is a passive tense, then the "knowledge of language" in the valence relation "dependent" is profiled, so it is translated into Chinese as following:

One is, what knowledge of language is needed for us to use language? (English)

其一, 什么样的语言知识才是我们使用语言所需要的? (Chinese)

Example 3: In a Sense, We Must Know a Language to Use it, but We Are not Always Fully Aware of This Knowledge

"In a sense" is a chunk expression. If it is unfamiliar to the learners, the translation process will be difficult. In the traditional dictionary, the word "sense" has dozens of meaning explanation with lots of items which is difficult for learners to choose the definition. The FrameNet corpus can describe the semantic frame of words and their valence relations, so the lexical meaning can be considered as a whole part, which improves the process of meaning identification. With the search in the corpus and comparing with the syntax realization, it reveals that the word "sense" triggers the frame "Linguistic meaning", and the valence relation is expressed as "in a [Meaning SENSE Target]". The learners have to consider the meaning constructing in a logical way, because it is different with the frame "Feeling" and frame "Opinion".

While the verb "know" has triggered four frames "Awareness", "Differentiation", "Certainty", and "Familiarity". According to the syntax realization and in the corpus and the logical reasoning of "be not aware of", the frame "Familiarity" is chosen. The verb "aware" triggers the frame "Awareness" and the syntax realization is always expressed with chunk expressions. All the words searched in the corpus are key words or the key elements in the semantic space, they are crucial for the meaning constructing during the translation process [7]. At the same time, the translation expression also has to consider the pragmatic and conceptual characters in the target language. This sentence can be translated as:

In a sense, we must know a language to use it, but we are not always fully aware of this knowledge. (English)

在一定意义上, 我们必须熟悉一门语言才能使用它, 但往往不能完全感知到 这种语言知识。(Chinese)

\section{Conclusion}

Meaning is the key element for the translation research [8], but the understanding of meaning is not relied on the formal transformation between languages, but on the concept of meaning constructing. It is a big challenge for the understanding of translation nature and the operation of translation teaching when dealing with abstract 
semantic transformation. With the background of big data, based on the cognitive FrameNet corpus with Frame Semantic theory and semantic constructing process, this article tries to analyze the semantic constructing characters in the mental space, frame concept, concept mapping, and conceptual integration, and present the valence relations of frame elements and logic processing combining with syntax realization of the grammatical function of frame element, types of phrases and syntax characters. As a result, the difficulty of the concept of asymmetric concepts, conceptual metaphor, a polysemy and other teaching difficulties in the translation teaching process can be solved in the operational level.

\section{References}

[1] E. Vyvyan, M. Green, Cognitive Linguistics: An Introduction, Edinburgh University Press, Edinburgh, 2006.

[2] F. Charles, Frame semantics, in: Linguistic Society of Korea (Eds.), Linguistics in the Morning Calm, Hanshin Publishing, Seoul, 1982, pp. 111-137.

[3] F. Charles, Scenes-and-frames semantics, in: A. Zampolli (Eds.), Linguistic Structures Processing, Amsterdam, North Holland, 1977, pp. 55-82.

[4] Zongying, Wang, Gaopan, Guo, Cognitive Aspect of Conceptual structure and the Translation of Specialty English, Shanghai Translation, 2 (2010) 33-36.

[5] M. Turner, Reading Minds: The Study of English in the Age of Cognitive Science, Princeton University Press, Princeton, 1991.

[6] L. Talmy, Toward a Cognitive Semantics, MIT Press, Cambridge, 2000.

[7] Guoqiang, Lu, A Contrastive Analysis of English-Chinese Conceptual Structures, Shanghai Foreign Language Education Press, Shanghai, 2008.

[8] R. T. Bell, Translation and Translating: Theory and Practice, Foreign Language Teaching and Research Press, Beijing, 2005. 